\title{
On the top-quark width measurement using a combination of resonant and non resonant cross sections.
}

\author{
Alexey Baskakov ${ }^{1, \star}$, Eduard Boos ${ }^{1,2, \star \star}$, and Lev Dudko ${ }^{1, \star \star \star}$ \\ ${ }^{1}$ Skobeltsyn Institute of Nuclear Physics M.V. Lomonosov Moscow State University, Moscow 119991, Rus- \\ sian Federation \\ ${ }^{2}$ Faculty of Physics M.V.Lomonosov Moscow State University Leninskie Gory, Moscow 119991, Russian \\ Federation
}

\begin{abstract}
Top quark width measurement is important and complex task. Most measurements are done in assumption of the SM top quark interactions. We would like to discuss model independent way of measuring the top quark width. Experimental accuracies of the top quark fiducial cross section measurements from $2 \%$ to $10 \%$ may allow to put constrains on the top quark width with $6 \%$ to $29 \%$ precision.
\end{abstract}

\section{Introduction}

The top quark was discovered more than 20 years ago [1,2] and it still remains the heaviest known elementary particle. This fact probably makes it the most promising window to the Beyond Standard Model (BSM) physics. The top quark parameters measurement is critical for testing deviations from the Standard Model (SM) predictions. During LHC operation period mass and inclusive cross sections were measured at the percentage level [3]. Indirect top quark width measurements in assumption of only SM decay modes have reached ten percent accuracy $[4,5]$. However direct measurements of the top quark width are far from that precision because of experimental resolution limitations of the top-quark mass spectrum [6]. Recent direct measurements presented by CMS and ATLAS collaborations are mostly limited by the jet energy scale and resolution [7,8]. To improve accuracy of direct measurements usage of the b-charge asymmetry in the s- and t-channels of the single top production was recently proposed [9].

In our paper we present first steps of putting model independent and fully gauge invariant limits on the top quark width using combinations of resonant and non resonant cross sections.

\section{Simulations}

Model-independent constrains on the Higgs boson width can be done from comparison of the on-/offshell regions. Importance of the off-shell region in Higgs production first time was mentioned in [10]. In paper [11], the method for deriving model-independent upper bound on the Higgs boson width

\footnotetext{
^e-mail: a.baskakov@cern.ch

$\star \star$ e-mail: boos@theory.sinp.msu.ru

$\star \star \star$ e-mail: lev.doudko@cern.ch
} 
from $p p \rightarrow Z Z, p p \rightarrow H \rightarrow Z Z$ events was proposed. These ideas can not be directly applied to the top quark. First, the Higgs is match more narrow resonance than the top quark, in other words $\frac{\Gamma_{H}}{m_{H}}<<\frac{\Gamma_{t}}{m_{t}}$. Second, in case of the Higgs, we can calculate separately amplitude for the pole, non pole and interference amplitude for $p p \rightarrow Z Z$ and $p p \rightarrow H \rightarrow Z Z$ contributions. In case of the top quark pair production with subsequent decay to $W b$ one can not separate contributions in gauge invariant way for the top pair and the single top production parts. Therefore we perform the computation of the complete gauge invariant set of diagrams and investigate sensitivity of different kinematic regions to deviations from the SM caused by the top quark width and related coupling. This approach allows to put model independent and fully gauge invariant constrains on the top quark width.

\subsection{Modeling details}

For simplicity we take into account only main signal events on the parton level for final states as the first step of our investigation. We model $g g \rightarrow t W^{-} \bar{b}$, when one top quark decays and another comes on its mass shell. Additional sub-processes with quarks in the initial state does not make significant impact on results since the gluon fusion gives up to $90 \%$ at LHC energies [13, 14]. Hadronization and fragmentation effects, as well as backgrounds impact are postponed to the next more realistic analysis. We use CompHEP generator [15] with MSTW2008 PDF [16] to generate events for the full gauge invariant diagram set Fig. 1 which include both single top quark and the top quark pair production. We take $S M$ values of the top quark mass and width $M_{t}^{S M}=172.5 \mathrm{GeV}, \quad \Gamma_{t}^{S M}=1.49 \mathrm{GeV}$. Process $g g \rightarrow$ $t W^{-} \bar{b}$ at tree level includes eight diagrams Fig. 1. We do not take into account NLO corrections since

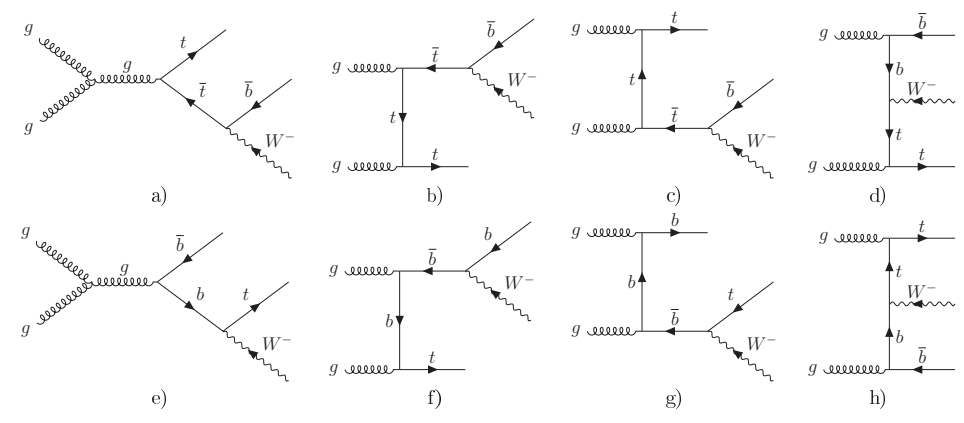

Figure 1. Full gauge invariant tree level diagram set for the $g g \rightarrow t W^{-} \bar{b}$ process.

their influence on the top quark width kinematic dependence is negligible. NLO QCD corrections to $W^{+} W^{-} b \bar{b}$ production at hadron colliders were calculated in [12]. We use notations shown in Eq. 1 and Eq. 2 to parametrize different kinematic regions of the $t W^{-} \bar{b}$ mass spectrum named as double pole (DP) and single pole (SP) respectively.

$$
\begin{gathered}
M_{t}^{S M}-n \cdot \Gamma_{t}^{S M} \leq M_{W-\bar{b}} \leq M_{t}^{S M}+n \cdot \Gamma_{t}^{S M} \\
M_{W^{-} \bar{b}} \leq M_{t}^{S M}-k \cdot \Gamma_{t}^{S M} \text { or } M_{t}^{S M}+k \cdot \Gamma_{t}^{S M} \leq M_{W^{-} \bar{b}}
\end{gathered}
$$

Parameters $n$ and $k$ are taken to be integer numbers that allow to change kinematic regions. They should fill the following relation $n \leq k$ as regions may not include the hole mass spectrum but should not overlap. The DP region determined in Eq. 1 and shown in Fig. 2 by the dark blue includes the areas around to the top quark mass peaks, one of the top is on shell in our simple example. In the SP 


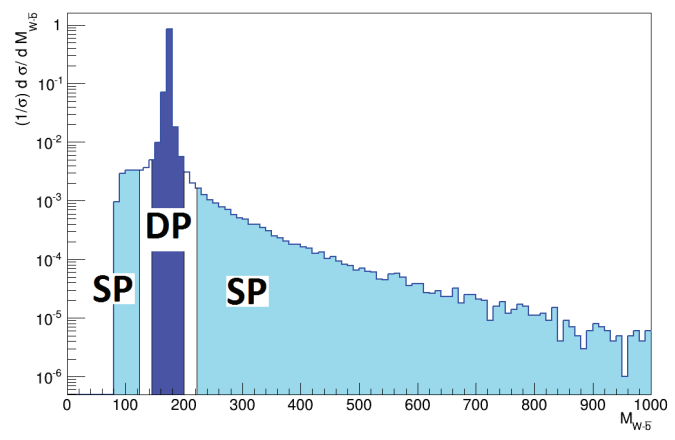

Figure 2. Differential mass spectrum of the $W^{-} \bar{b}$. Double pole(DP) corresponds to the $t \bar{t}$ kinematic region Eq. 1, single pole(SP) corresponds to the single top quark region Eq. 2.

regions (Eq. 2) shown by light blue in Fig. 2 one of $W b$ invariant mass is close to the top mass shell (on the mass shell in our example) while the second invariant mass is out of the top pole aria. $\Gamma_{t}^{S M}$ is a constant, in its units it is shown how far from the top quark pole the $W b$ invariant mass is taken.

\subsection{Top quark width parametrization}

For the top quark width parametrization we use following notations

$$
\Gamma_{t}=\xi^{2} \cdot \Gamma_{t}^{S M}+\Delta,
$$

reflecting the fact that the top quark width might be different from the SM width either by a modification of the Wtb coupling or by an existence of some non-SM additional decay modes [17-21]. In Eq. 3 the parameter $\xi$ is used to reweight simultaneously the top quark width and the Wtb coupling, note that the production cross section times the branching ratio remains unchanged. It is useful to parametrize the deviation $\Delta$ also in terms of the SM width as $\Delta=\delta \cdot \Gamma_{t}^{S M}$. Details are given in Appendix 5. The invariant $W^{-} \bar{b}$ mass distribution for different values of $\xi$ and $\delta$ is shown in Fig. 3. The
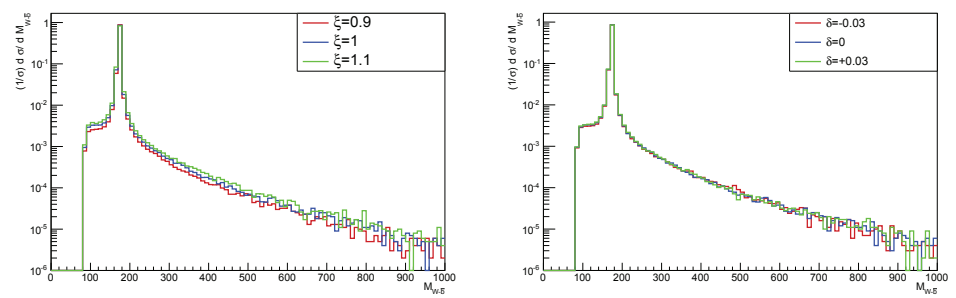

Figure 3. Differential mass spectrum of the $W^{-} \bar{b}$ for different $\xi$ and $\delta$.

influence of the $n$ and $k$ parameter variation on the cross section in DP and SP regions is demonstrated in Fig. 4. We see that DP region loose sensitivity to $\xi$ variation for $n \geq 15$ Fig. 4 (top left) and influence of $\delta$ parameter keeps valuable for all $n$ values Fig.4 (bottom left). In contrast for the SP region the sensitivity to $\xi$ variation is visible for all physically meaningful $k$ values, while $\delta$ modification dose not make any influence. This observation allows to put constrains on the $\xi$ and $\delta$ using two dimension scan over the $n$ and $k$ parameters. 

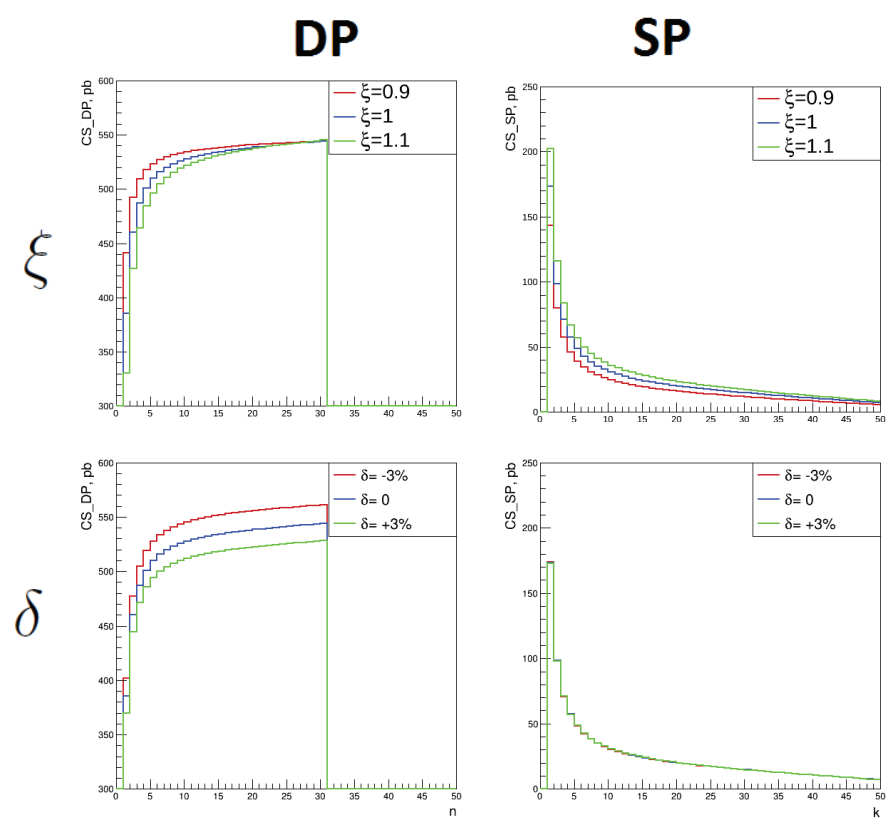

Figure 4. Influence of $n$ and $k$ parameters variation on the cross section in DP and SP regions.

\subsection{Cross section parametrization}

Now the cross sections in DP and SP regions can be parametrized in terms $\xi$ and $\delta$. Current experimental data [4-8] indicate that the deviations from the SM for the top width should be small. To keep deviations on both $\xi$ and $\delta$ small let us make the following substitution

$$
\epsilon=\xi^{2}-1
$$

and perform the linear expansion of the fiducial cross sections with respect to small parameters $\epsilon$ and $\delta$ as follows:

$$
\begin{gathered}
\sigma_{D P}=\sigma_{D P}^{S M}+A_{D P} \cdot \epsilon+B_{D P} \cdot \delta \\
\sigma_{S P}=\sigma_{S P}^{S M}+A_{S P} \cdot \epsilon+B_{S P} \cdot \delta
\end{gathered}
$$

$A, B$ are numerically calculated from DP and SP regions for different $n, k$.

Using the standard $\chi^{2}$ method $\left(\chi^{2}(\sigma)=\left(\frac{\sigma^{S M}-\sigma}{\Delta \sigma}\right)^{2}\right)$ and requiring that the SM and modified cross sections are within two standard deviations from each other, we derive an upper limits on $\epsilon$ and $\delta$ at the $95 \%$ confidence level. Figures 5, 6 show fits for different expected experimental measurements accuracies of 2,5 or 10 . As it was mentioned in 2.2, DP region has sensitivity mostly to $\delta$ parameter, while SP region is more sensitive to $\epsilon$ (or $\xi$ see Eq. 4). Choice of the kinematic region boundary by varying $n$ and $k$ parameters make some shifts on the plots, but do not change the general dependence for each region. This fact allows to put much stronger combined limits on $\delta$ and $\epsilon$ Fig. 7, were two combinations $n=k=15$ and $n=15, k=30$ are shown. We use Eq. 3 and take obtained parameters limits in quadratures to get predictions on the top quark width measurement precision Tab. 1. 
DP fit, uncertainty of $10,5,2 \%$
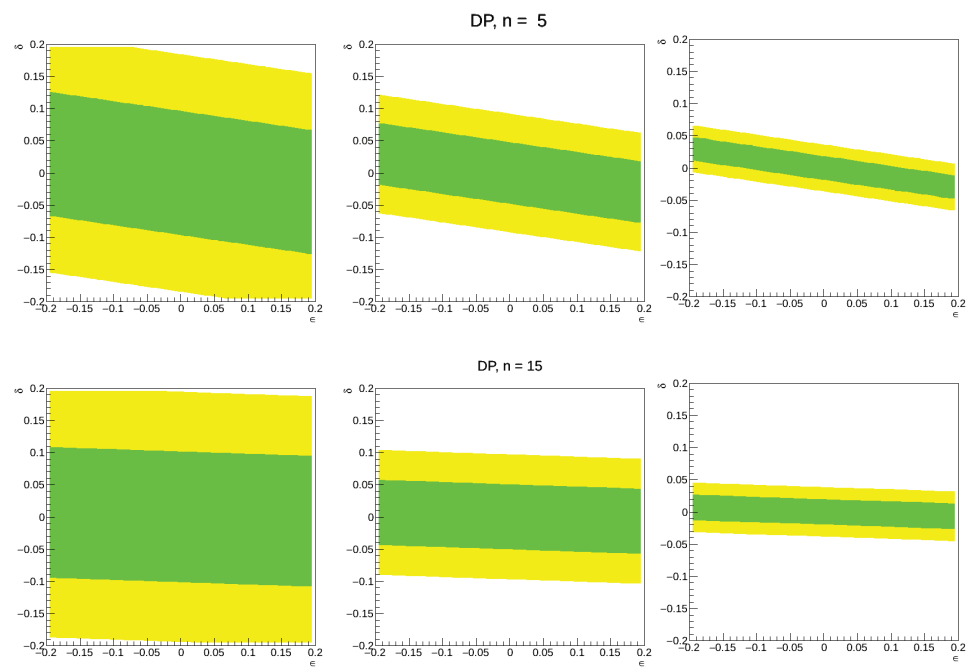

Figure 5. Constraints on the $\epsilon$ and $\delta$ parameters from DP region for boundary of \pm 5 (top row) and \pm 15 (bottom row) $\Gamma_{t}^{S M}$ from the top quark mass value. Cross section experimental measurements accuracies are taken 10,5 and $2 \%$ (from left to right). Green and yellow arias correspond to exclusion limits at $68 \%$ and $95 \%$ CL on $\epsilon$ and $\delta$.

SP fit, uncertainty of $10,5,2 \%$
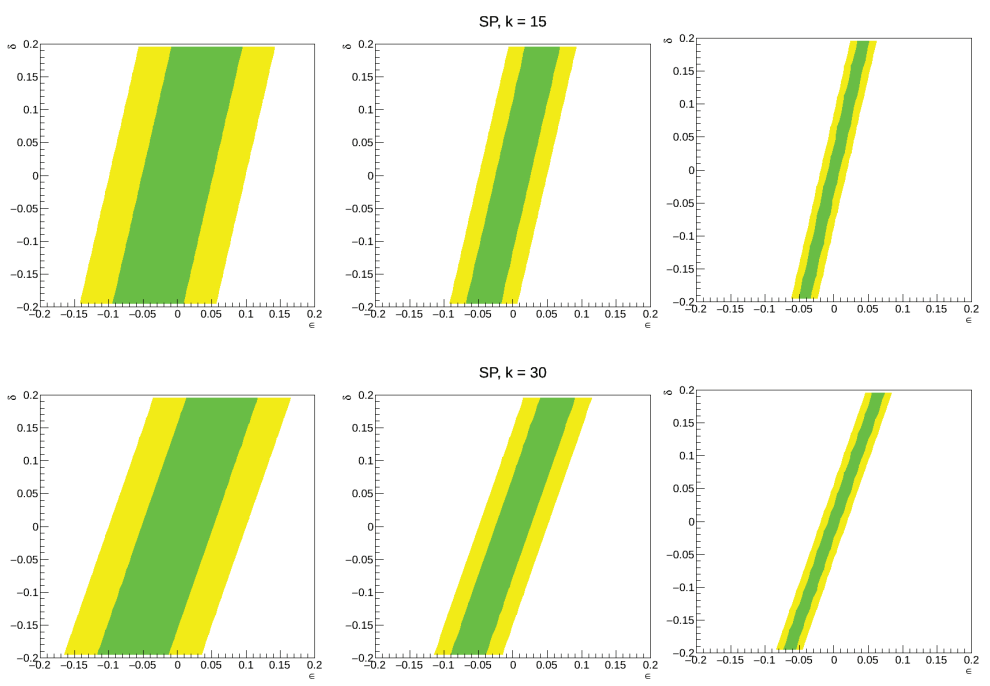

Figure 6. Constraints on the $\epsilon$ and $\delta$ parameters from SP region for $k=15$ (top row) and $k=30$ (bottom row). Cross section experimental measurements accuracies are taken 10,5 and 2\% (from left to right). Green and yellow arias correspond to exclusion limits at $68 \%$ and $95 \% \mathrm{CL}$ on $\epsilon$ and $\delta$. 
Combined fit, uncertainty of $10,5,2 \%$
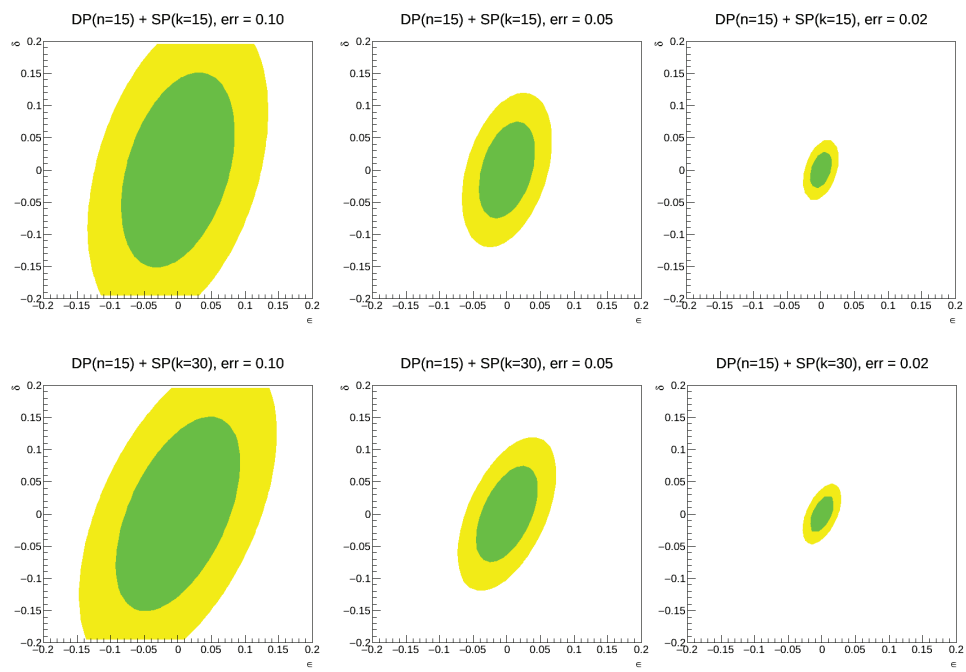

Figure 7. Limits on the $\epsilon$ and $\delta$ parameters obtained from DP and SP regions simultaneously. Region boundaries are taken as $n=k=15$ (top row) and $n=15, k=30$ (bottom row). Green and yellow arias correspond to exclusion limits at $68 \%$ and $95 \%$ CL on $\epsilon$ and $\delta$.

\begin{tabular}{|c|c|}
\hline Experimental CS accuracy & Obtained limit on the top quark width \\
\hline $2 \%$ & $\geq 6 \%$ \\
\hline $5 \%$ & $\geq 14 \%$ \\
\hline $10 \%$ & $\geq 29 \%$ \\
\hline
\end{tabular}

Table 1. Estimations on achievable constrains on the top quark width for different experimental accuracies of the top quark fiducial cross section measurement.

\section{Results}

Gauge invariant estimation of deviations of the top quark width from its SM value is obtained in different kinematic regions. It is shown that top quark production cross section in the Double Pole(DP) region is more sensitive to $\delta$, while in the Single Pole(SP) to $\epsilon(\xi)$ parameter. This fact allows to put combined limits on both parameters simultaneously and using these limits obtain constrains on the top quark width. Achievable constrains on the model independent top quark width measurement are estimated to be $6 \%$ to $29 \%$ for corresponding experimental accuracies from $2 \%$ to $10 \%$. This results are achieved using simplified model, all effects as hadronization and fragmentation, detector response and background events will be taken into account in the following study.

\section{Acknowledgments}

The work was supported by grant 16-12-10280 of Russian Science Foundation. 


\section{References}

[1] Abe, F. et al. (CDF Collaboration), Observation of top quark production in $\bar{p} p$ collisions, Phys. Rev. Lett. 74, 2626 (1995).

[2] S. Abachi et al. (D0 Collaboration), Observation of the top quark, Phys. Rev. Lett. 74, 2632 (1995).

[3] C. Patrignani et al. (Particle Data Group), Chin. Phys. C40, 100001 (2016).

[4] V. M. Abazov et al. (D0 Collaboration), Improved determination of the width of the top quark, Phys. Rev. D85, 091104 (2012).

[5] CMS collaboration, "Measurement of the ratio $\mathcal{B}(t \rightarrow W b) / \mathcal{B}(t \rightarrow W q)$ in pp collisions at $\sqrt{s}=$ 8 TeV", Phys. Lett. B736, 33-57 (2014).

[6] CDF Collaboration, "Direct Measurement of the Total Decay Width of the Top Quark", Phys. Rev. Lett. 111, 202001, (2013).

[7] CMS Collaboration, "Bounding the top quark width using final states with two charged leptons and two jets at $\sqrt{s}=13 \mathrm{TeV} ",(2016)$.

[8] ATLAS collaboration, "Direct top-quark decay width measurement in the $t \bar{t}$ lepton+jets channel at $\sqrt{s}=8 \mathrm{TeV}$ with the ATLAS experiment" ATLAS-CONF-2017-056, (2017).

[9] P.P. Giardino, C. Zhang, Phys. Rev. D96, 011901 (2017).

[10] N. Kauer and G. Passarino, JHEP 1208, 116 (2012).

[11] F. Caola, K. Melnikov, Phys. Rev.D88, 054024 (2013).

[12] A. Denner, S. Dittmaier, S. Kallweit, S. Pozzorini, Phys. Rev. Lett.106, 052001 (2011).

[13] S. Moch and P. Uwer, Phys.Rev. D 78, 034003 (2008).

[14] K. Olive et al., Particle Data Group Collaboration, Chin.Phys. C 38,090001 (2014).

[15] E.E. Boos, V.E. Bunichev, L.V. Dudko, V.I. Savrin, A.V. Sherstnev, Phys.Atom.Nucl. 69, 1317 (2006).

[16] A.D. Martin, W.J. Stirling, R.S. Thorne et al., Eur. Phys.J. C 63, 189 (2009).

[17] W. Bernreuther, J. Phys. G35, 083001 (2008).

[18] F. Larios and R. Martinez and M. A. Perez, Int. J. Mod. Phys. A21, 3473-3494 (2006).

[19] T. Han, K. Whisnant, B.L. Young and X. Zhang, Phys. Rev. D55, 7241-7248 (1997).

[20] M.J. Herrero, 29th International Meeting on Fundamental Physics Barcelona, Spain (2011).

[21] A. Johan. R. Frederix, J. -M. Gerard, A. Giammanco, M. Herquet, S. Kalinin, E. Kou, V.

Lemaitre and F. Maltoni, F. Eur. Phys. J. C49, 791-801 (2007).

[22] N. Kauer, D. Zeppenfeld, Phys. Rev. D65, 014021 (2001).

\section{Appendix}

The general answer for the Breit-Wigner type integral has the following form

$$
I_{B W}=\int_{q_{\max }^{2}}^{q_{\min }^{2}} \frac{d q^{2}}{\left(q^{2}-M^{2}\right)^{2}+\Gamma^{2} M^{2}}=\frac{1}{\Gamma M}\left[\operatorname{arctg}\left(\frac{q_{\max }^{2}}{M \Gamma}-\frac{M}{\Gamma}\right)-\operatorname{arctg}\left(\frac{q_{\min }^{2}}{M \Gamma}-\frac{M}{\Gamma}\right)\right] .
$$


If we define different kinematic regions as in Fig. 8, then $I_{1}$ can be interpreted as DP region Eq. 1 and $I_{2}+I_{3}$ as SP region Eq. 2. In the limit $\Gamma<<M$ and $\Delta M^{ \pm}<M$

$$
\begin{gathered}
I_{1}=\frac{\pi}{M \Gamma}-\frac{1}{2 M}\left(\frac{1}{\Delta M^{+}}-\frac{1}{\Delta M^{-}}\right)+\ldots \\
I_{2}=\frac{1}{2 M}\left(\frac{1}{\Delta M^{+}}-\frac{1}{M}-\frac{2 M}{M_{c u t}^{+}-M^{2}}+\ldots\right)+\ldots \\
I_{3}=\frac{1}{2 M}\left(\frac{1}{\Delta M^{-}}+\frac{1}{M}-\frac{2 M}{M^{2}-M_{\text {cut }}^{-}}+\ldots\right)+\ldots
\end{gathered}
$$

The Equation 8 allows to understand qualitatively observed properties in Fig. 4 if one takes into

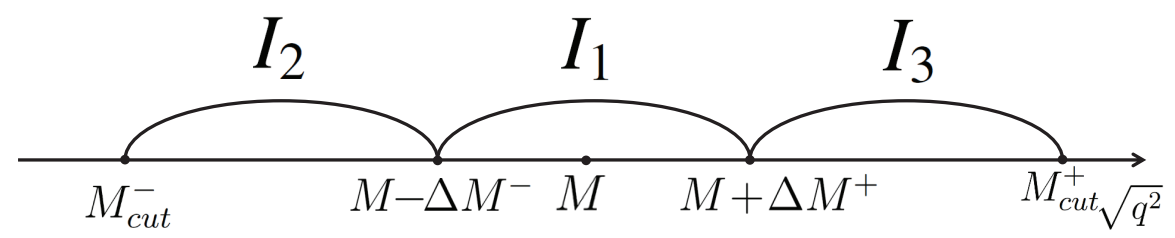

Figure 8. Definition of kinematic regions.

account the dependence of the top quark width on both parameters $\epsilon$ and $\delta$ and the fact that the numerator of the matrix element squared depends only on the parameter $\epsilon$ via the Wtb coupling. Furthermore, one can see that if $M_{c u t}^{-} \rightarrow-\infty$ and $M_{c u t}^{+} \rightarrow+\infty$

$$
I_{B W}=I_{1}+I_{2}+I_{3}=\frac{\pi}{M \Gamma} .
$$

At $\Delta M^{+}=\Delta M^{-}=n \cdot \Gamma$ we get formula (19) [22]

$$
I_{1}=\frac{\pi}{M \Gamma}\left(1-\frac{1}{n \Gamma}+\ldots\right) .
$$

For our consideration this formula (19) [22] is not useful since we want to study dependence on $\Gamma$, but $\Delta M^{+}$and $\Delta M^{-}$are cuts and do not depend on $\Gamma$. 